\title{
Ocular abnormalities in a large patient cohort with retinitis pigmentosa in Western China
}

\author{
Lian Tan ${ }^{1,2}$, Yanling Long ${ }^{1,2}$, Ziyang Li ${ }^{1,2}$, Xi Ying ${ }^{1,2}$, Jiayun Ren ${ }^{1,2}$, Cheng Sun ${ }^{1,2}$, Xiaohong Meng ${ }^{1,2^{*}}$ and
} Shiying $\mathrm{Li}^{1,2^{*}}$ (D)

\begin{abstract}
Background: To report the prevalence of ocular abnormalities and investigate visual acuity in a large cohort of retinitis pigmentosa (RP) patients in Western China.

Methods: The medical records and ophthalmic examination reports of 2127 eyes of 1065 RP patients at one eye hospital were retrospectively reviewed to determined the prevalence of ocular abnormalities and the relationship between best corrected visual acuity (BCVA) and macular abnormalities.

Results: Nyctalopia (58.2\%) and blurred vision (27.1\%) were the leading reasons for RP patients to request an ophthalmic examination. BCVA measurements in the better eyes at first clinical presentation showed that 304 patients (28.5\%) were categorised as blind and 220 patients (20.7\%) as low vision. The most common ocular abnormalities were macular abnormalities (59.7\%) and cataracts (43.1\%). The macular abnormalities included epiretinal membranes (51.1\%), cystoid macular edema (18.4\%), vitreomacular traction syndrome (2.4\%), macular holes (2.3\%) and choroidal neovascular membranes (0.05\%). Glaucoma was found in 35 eyes (1.6\%). The proportions of epiretinal membranes $(p=0.001)$ and macular holes $(p=0.008)$ increased significantly with age. Cystoid macular edema was significantly associated with poorer visual acuity in RP patients with clear lens $(p=0.002)$.

Conclusion: Cataracts and macular abnormalities are common in RP patients. Of the macular abnormalities, cystoid macular edema may have a negative effect on BCVA in RP patients with clear lens. Therefore, optical coherence tomography screening in RP patients is highly recommended for early detection and treatment of maculopathy.
\end{abstract}

Keywords: Retinitis pigmentosa, Ocular abnormalities, Macular abnormalities, Cataract, Glaucoma, Best corrected visual acuity

\section{Background}

Retinitis pigmentosa (RP) is the most common type of inherited retinal dystrophy, causing progressive degeneration of the retinal pigment epithelium (RPE) and photoreceptors [1]. RP prevalence is approximately $1 / 4000$

\footnotetext{
*Correspondence: cqwmwm@163.com; shiying_li@126.com

'Southwest Eye Hospital/Southwest Hospital, Third Military Medical University (Army Medical University), Chongqing 400038, China

Full list of author information is available at the end of the article
}

and more than 1.5 million patients are affected worldwide [2]. Nyctalopia and blurred vision are the most common RP symptoms, but other rare symptoms (e.g. photophobia, metamorphopsia) also prompt RP patients to visit a doctor [1-3]. However, little systematic information has been published on the clinical symptoms that RP patients experience before diagnosis.

Ocular abnormalities, such as glaucoma, cataracts, maculopathy, etc., may occur as RP progresses [4]. The typical histopathological change in RP is thinning of the

(c) The Author(s). 2021 Open Access This article is licensed under a Creative Commons Attribution 4.0 International License, which permits use, sharing, adaptation, distribution and reproduction in any medium or format, as long as you give appropriate credit to the original author(s) and the source, provide a link to the Creative Commons licence, and indicate if changes were made. The images or other third party material in this article are included in the article's Creative Commons licence, unless indicated otherwise in a credit line to the material. If material is not included in the article's Creative Commons licence and your intended use is not permitted by statutory regulation or exceeds the permitted use, you will need to obtain permission directly from the copyright holder. To view a copy of this licence, visit http://creativecommons.org/licenses/by/4.0/ The Creative Commons Public Domain Dedication waiver (http://creativecommons.org/publicdomain/zero/1.0/) applies to the data made available in this article, unless otherwise stated in a credit line to the data. 
photoreceptors' outer segments, which worsens as RP progresses $[4,5]$. Although central vision acuity can remain normal for several years, anatomical macular abnormalities may occur in early-stage RP [6, 7]. The most common macular abnormalities in RP patients are epiretinal membrane (ERM) and cystoid macular edema (CME), which are detected by optical coherence tomography (OCT). Other macular abnormalities also accompanied by, such as macular holes $(\mathrm{MH})$, vitreomacular traction syndrome (VMT) and choroid neovascularisation membrane (CNVM) [5-9]. To the authors' knowledge, visual acuity and prevalence of ocular abnormalities have not been reported previously in a large cohort of RP patients in Western China.

Therefore, this study assessed ocular abnormalities in a large cohort of RP patients in Western China and investigated correlations between visual acuity and macular abnormalities.

\section{Methods}

\section{Study design and subjects recruitment}

The authors retrospectively extracted medical records of patients diagnosed with RP between January 2014 and January 2019 at Southwest Hospital/ Southwest Eye Hospital, Third Military Medical University (Army Medical University), Chongqing, China. The records included information on each patient's age, sex, medical and surgical history, family history, complaints, best corrected visual acuity (BCVA), intraocular pressure, lens status and the slit-lamp anterior segment and dilated fundus examination from the first clinical presentation. RP diagnosis was based on: (1) presence of night blindness or blurred vision and peripheral visual field restriction; (2) characteristic fundus changes, such as pale optic disc, attenuated vessels and bone-spicule-like pigmentation deposits in the midor far-periphery; and (3) reduced or non-detectable fullfield electroretinogram (ff-ERG) rod and cone amplitudes $[1,4,5]$. The exclusion criteria were: (1) a history of trauma; (2) a history of vitreoretinal surgery and intravitreal therapy; (3) pathological myopia; (4) other vascular retinopathies, such as hypertensive retinopathy, diabetic retinopathy, retinal periphlebitis, etc.; (5)age-related macular degeneration; (6) atypical RP, such as unilateral pigmentary retinopathy or sectorial pigmentary retinopathy; (7) secondary retinal pigmentosa; and (8) severe systemic diseases. The study was performed according to the Declaration of Helsinki and approved by the Ethics and Research Committee of Southwest Hospital, Army Medical University (KY2020096).

\section{Age of onset and functional examination}

The age of onset (that is, of symptoms) was defined as the patient's age subtracted from the year with a positive disease history. BCVA was measured with a Tumbling E chart and converted into the logarithm of the minimum angle of resolution (logMAR) value for analysis [10]. BCVA was classified according to the World Health Organization's (WHO) category of vision as follows [2]: BCVA worse than $3 / 60$ in the better eye was classified as blindness; BCVA of $3 / 60-6 / 18$ in the better eye was classified as low vision; and BCVA of $6 / 18$ or more was classified as normal. The researchers did not classify visual acuity according to the visual field. Full-field electroretinogram testing was performed according to the International Society of Clinical Electrophysiology of Vision's standards [11].

\section{Imaging examination}

The lens condition was classified as clear, cataract, pseudophakic or aphakic. A specialist diagnosed glaucoma based on the presence of glaucomatous optic neuropathy and intraocular pressure over $21 \mathrm{mmHg}$, with or without the presence of iridotrabecular contact [12]. The macular microstructure was examined with Spectral Domain OCT (SD-OCT) (Cirrus HD-OCT, Carl Zeiss Meditec, Dublin California, USA) or Heidelberg Spectralis OCT (Heidelberg Engineering, Heidelberg, Germany). Two experienced ophthalmologists independently evaluated the images. If the results differed, a third ophthalmologist re-evaluated them. The macular abnormalities were documented as: ERM, CME, MH (including lamellar and full-thickness $\mathrm{MH}$ ), VMT and CNVM according to the following definitions $[1,13,14]$ :

ERM was diagnosed with the presence of as avascular, fibrocellular membrane on the inner surface of the retina, often resulting from proliferative changes at the vitro-retinal interface.

CME was defined as the presence of cystoid spaces, appearing like small hypo-reflective lacunae with welldefined boundaries on two or more consecutive views of the radial scan in the macular area.

Lamellar-thickness macular hole (LMH) was defined as partial thickness defects of the macular area, with an irregular foveal contour and a schisis between the inner and the outer retinal layers and without any photoreceptor layer defects. Full-thickness macular hole (FTMH) was defined as a vertical split in the neurosensory layers of the foveal region.

VMT was characterized by a vitromacular adhesion that involved the foveal region from posterior hyaloid face, causing traction and distortion of the central macular.

CNVM was defined as cystic macular edema associated with a disruption of the Bruch membrane/retinal pigment epithelium (RPE) complex, accompanied by an avascular structure emanating from the deep capillary plexus and appearing as a hyperreflective lesion connected with the subretinal pigment epithelium. Other 
diseases that cause macular CNVM were excluded [9, 14].

\section{Molecular diagnosis and inheritance pattern}

Some patients voluntarily underwent molecular diagnosis, and inheritance patterns were categorised according to the genetic test reports: autosomic dominant (AD), autosomic recessive (AR), X-linked (X-L) or sporadic (i.e., patients with negative genetic reports or no evidence of other affected family members). RP patients' ages were divided into four groups for statistical analysis ( $\leq 15$ years, $16-44$ years, $45-64$ years and $\geq 65$ years).

\section{Statistical analyses}

SPSS 22.0 was used to conduct analyses. Continuous variables, such as counselling age, age of onset and BCVA (logMAR), were expressed as means \pm standard deviation (SD) and were compared with independent sample t-tests. Categorical variables (sex, complaints, inheritance pattern, age group, lens condition and macular abnormalities) were presented as counts and percentages and compared with Chi-squared or Fisher's exact tests. Multiple linear regression investigated the relationship between BCVA (logMAR) and macular abnormalities. Coefficients of the estimated regression $(\beta)$, the corresponding statistical significance $(P)$, the exponential parameter and its confidence interval were presented for each factor. A $P$-value of $<0.05$ was considered statistically significant.

\section{Results}

A total of 2127 eyes belong to 1065 patients (493 [46.3\%] female and 572 [53.7\%] male, respectively.) were investigated. Table 1 describes the patients' demographic characteristics. The number of eyes did not match the number of patients because three eyeballs in three female patients had been enucleated due to glaucoma. The

Table 1 Demographic characteristics of the 1065 RP patients in this study

\begin{tabular}{llll}
\hline & Female & Male & Total \\
\hline No. patients & $493(46.3 \%)$ & $572(53.7 \%)$ & $1065(100 \%)$ \\
No. Eyes & $983^{\mathrm{a}}(45.2 \%)$ & $1144(53.8 \%)$ & $2127(100 \%)$ \\
Counseling Age (yrs) & $43.4 \pm 16.0$ & $40.6 \pm 15.3$ & $41.9 \pm 15.7$ \\
Mean age of onset (yrs) & $24.1 \pm 19.7$ & $20.0 \pm 19.6$ & $21.9 \pm 19.2$ \\
Inheritance pattern & & & \\
$\quad$ Autosomic dominant (No.) & $14(8.6 \%)$ & $17(9.0 \%)$ & $31(8.8 \%)$ \\
$\quad$ Autosomic recessive (No.) & $99(60.7 \%)$ & $104(55.0 \%)$ & $203(57.7 \%)$ \\
$\quad$ X-linked (No.) & $3(1.9 \%)$ & $18(9.5 \%)$ & $21(6.0 \%)$ \\
$\quad$ Sporadic (No.) & $47(28.8 \%)$ & $50(26.5 \%)$ & $97(27.6 \%)$ \\
$\quad$ Total (No.) & $163(100 \%)$ & $189(100 \%)$ & $352(100 \%)$ \\
\hline a: Three eyeballs in three female patients were enucleated due to glaucoma
\end{tabular}

mean \pm SD counselling age at the patient's first eye hospital visit was $41.9 \pm 15.7$ years (range: 3 to 83 years; females: $43.4 \pm 16.0$; males: $40.6 \pm 15.3 ; p=0.000$ ). The mean \pm SD age of onset of symptoms for RP patients was $21.9 \pm 19.2$ years (females: $24.1 \pm 19.7$; males: $20.0 \pm$ 19.6; $p=0.000)$. A total of 352 of 1065 patients $(33.1 \%)$ had a molecular diagnosis, and the most common inheritance pattern was AR (57.7\%), followed by sporadic (27.6\%), AD (8.8\%) and X-L (6.0\%) (Table 1).

Nyctalopia (58.2\%) and blurred vision (27.1\%) were the main visual complaints (Fig. 1). Among the patients' in the study, $11.2 \%$ had experienced poor vision since childhood ( $\leq 15$ years old). Other reasons for RP patients visiting the hospital included routine physical examination (1.1\%), metamorphopsia (0.7\%), photophoby $(0.5 \%)$, black floating spots $(0.5 \%)$ and other unusual symptoms $(0.7 \%$, including pain, photopsia, a narrow visual field and double vision).

The BCVA values in the better eye at first clinical presentation showed that $304(28.5 \%)$ patients were blind, and 220 (20.7\%) had low vision (Fig. 2a; Supplemental Table 1). Although the percentage of normal vision at first presentation in females (53.1\%) was slightly higher than in males (48.8\%), no significant sex difference was observed in visual acuity distribution $(p=$ 0.283). Patients over the age of 44 showed a lower proportion (45-64y: $45 \%$; $\geq 65 \mathrm{y}: 31.5 \%$ ) of normal vision than patients under 44 ( $\leq 15 \mathrm{y}: 59.0 \%$; $16-44 \mathrm{y}: 57.6 \%)$ at first presentation (Fig. 2b; Supplemental Table 2), and the proportion of blindness in patients over 44 years (45-64y: 34.6\%; $\geq 65 y$ : 43.8\%) was higher than in patients under 44 years ( $\leq 15 \mathrm{y}: 8.5 \% ; 16-44 \mathrm{y}: 14.8 \%)$. There was also a significant increase in the percentage of blindness with age (Fig. 2b; $p=0.000$ ).

Cataracts were observed in 917 eyes $(43.1 \%, 917 /$ 2127 eyes) of 469 patients in the sample $(44.0 \%, 469 /$ 1065 patients). Pseudophakic and aphakic eyes were classed as presenting cataracts: of the 917 eyes in which cataracts were observed, pseudophakia was seen in 157 eyes $(7.4 \%)$ of 95 patients (4.5\%), and aphakia was seen in 22 eyes (1.0\%) of 14 patients $(0.7 \%)$. Glaucoma was found in 35 eyes $(1.6 \%$; 35/2127) of 21 patients $(2.0 \%$; 21/1065). Macular OCT was performed in 1388 eyes $(65.3 \% ; 1388 / 2127)$ of 704 patients (66.1\%; 704/1065), and macular abnormalities were seen in 829 eyes $(59.7 \% ; 829 / 1388)$ of 481 patients $(68.3 \%$; 481/704).

Typical OCT and corresponding fundus photography of macular abnormalities in RP patients are shown in Fig. $3(\mathrm{a}-\mathrm{j})$. The prevalence of macular abnormalities was as follows:

- ERM: 709 eyes (51.1\%; 709/1388) of 418 patients (59.4\%; 418/704). 


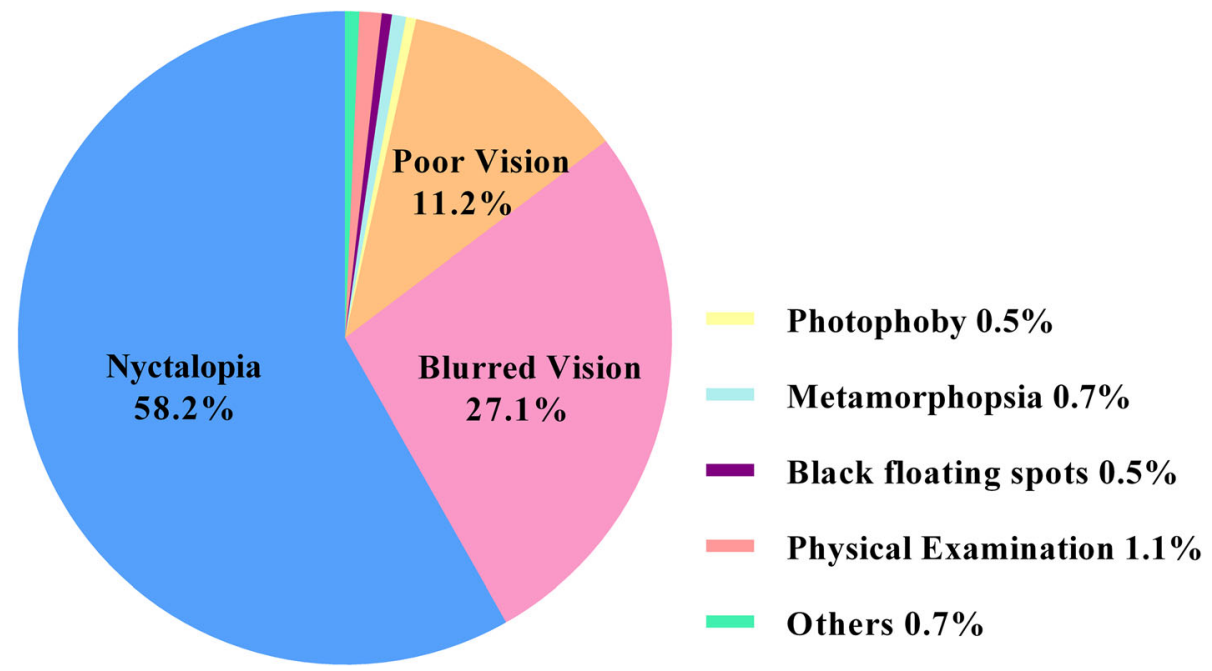

Fig. 1 Distribution of chief complaints in the study cohort of patients with RP. The pie diagrams showed the sampled patients' complaint were distributed as follows: nyctalopia (58.2\%), blurred vision (27.1\%), poor vision since childhood ( $\leq 15$ years old, 11.2\%), physical examination (1.1\%), metamorphopsia (0.7\%), photophoby (0.5\%), black floating spots $(0.5 \%)$ and other unusual symptoms $(0.7 \%$, including pain, photopsia, a narrow vision field and double vision)

- CME: 255 eyes (18.4\%; 255/1388) of 150 patients (21.3\%; 150/704).

- VMT: 33 eyes $(2.4 \% ; 33 / 1388)$ of 25 patients $(3.6 \%$; 25/704).

- $\mathrm{MH}: 32$ (2.3\%; $32 / 1388$ eyes) of 26 patients (3.7\%; 26/704).

- CNVM in one eye $(0.05 \% ; 1 /, 2127)$ of one female patient $(0.09 \% ; 1 / 1065)$.

Supplemental Tables 3, 4, 5 present the macular abnormality frequencies (stratifying patients according to sex, age and lens status) and show the corresponding statistical analysis. The results showed no significant differences among the classifications of macular abnormalities and sex (Fig. 4a, CME: $p=0.193$; VMT: $p=0.176$; MH: $p=0.383$ ), except for ERM (males: 55.7\%; females: $46.3 \% ; p=0.006$ ).

$\mathrm{MH}$ and VMT were not found in any patients $\leq 15$ years old. ERM $(p=0.001)$ and $\mathrm{MH}(p=0.008)$ were significantly more prevalent in older RP patients, and prevalence increased with age (Fig. 4b; Supplemental Table 4). No differences were observed in the

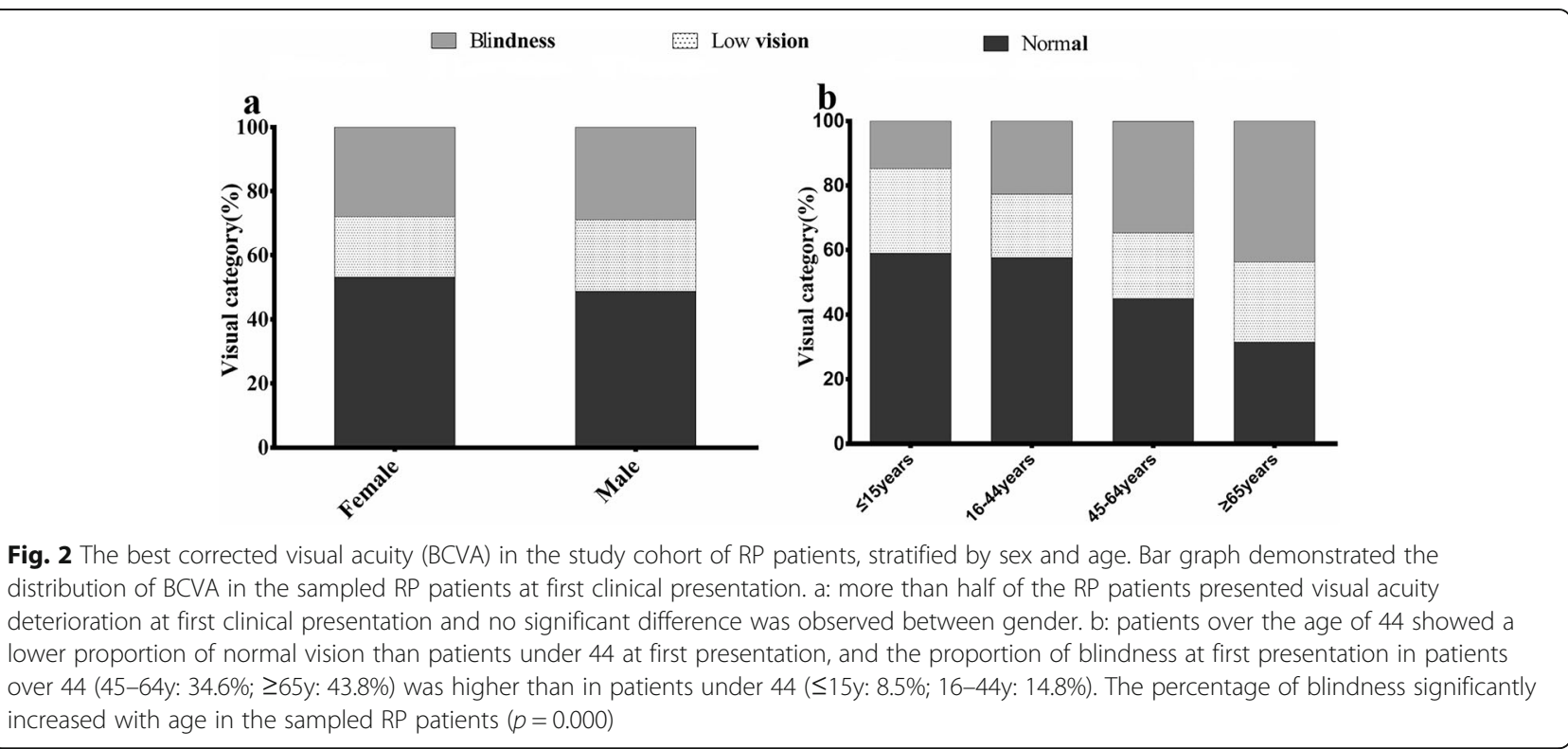




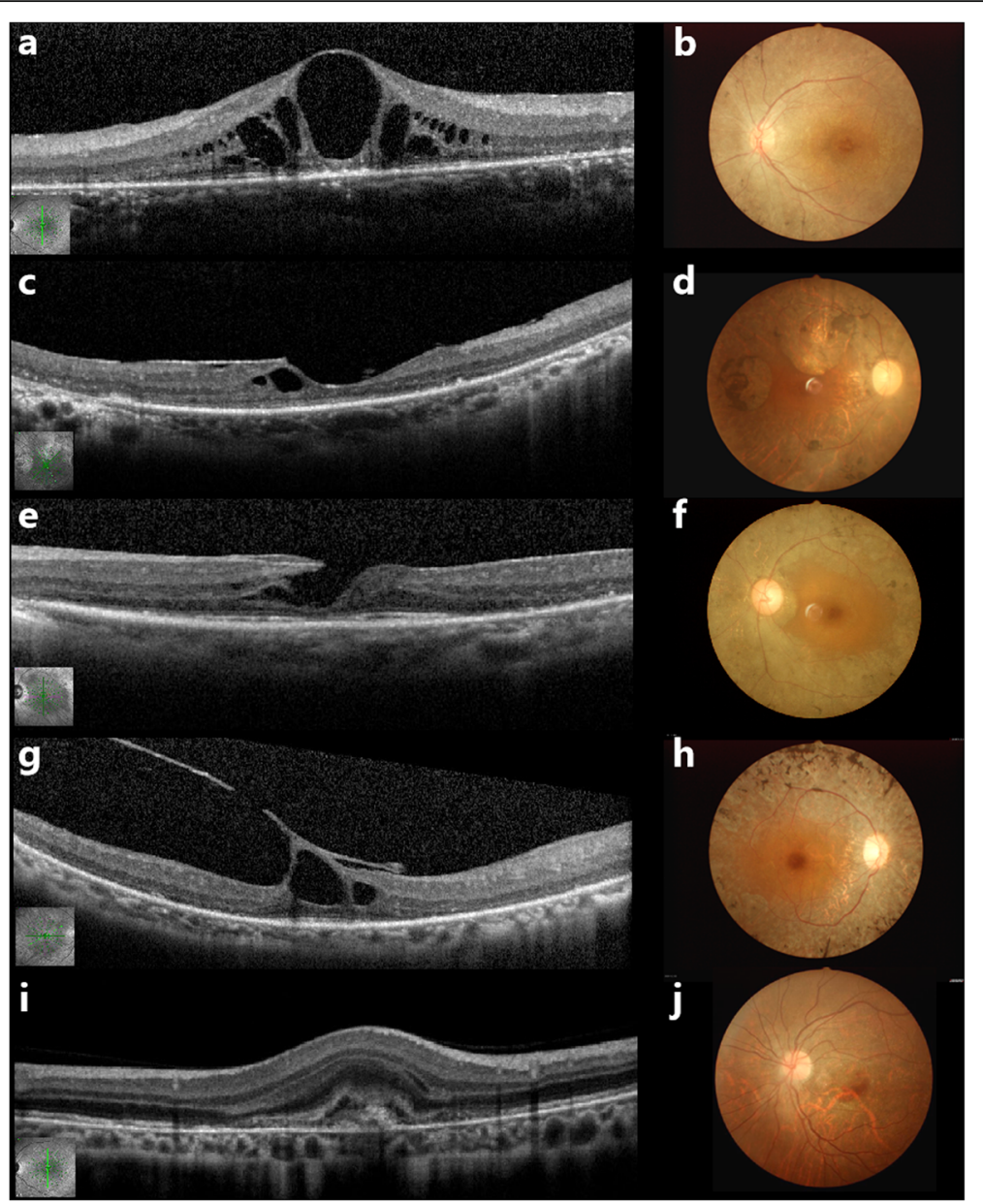

Fig. 3 Representative optical coherence tomography (OCT) and corresponding fundus photography of RP patients with macular abnormalities. a An OCT image with cystic-appearing spaces in the left eye. b Fundus photograph of the left eye in picture a, showing bone spicule pigmentation in the mid-periphery and vessels attenuation. c OCT scan showed a homogenous layer of moderately reflective material, present on the inner retinal layer. $\mathbf{d}$ Fundus photography of the right eye in picture $\mathrm{c}$, showed marked bone spicule pigmentation in the mid-periphery, waxy pallor of the optic disc and attenuated vessels. e OCT scan with lamellar macular hole. f: Fundus photography of the picture e, revealed bone spicule pigmentation in the mid-periphery and vessels attenuation. $\mathbf{g}$ OCT scan showed vitreomacular traction. $\mathbf{h}$ Fundus photography of the right eye in picture g, showed marked bone spicule pigmentation in the mid-periphery, waxy pallor of the optic disc and attenuated vessels. $\mathbf{i}$ OCT image showed disruption of the Bruch membrane/retinal pigment epithelium complex, accompanied by a hyper-reflective lesion connected with the subretinal pigment epithelium. $\mathbf{j}$ Fundus photography of the left eye in picture i, showed hemorrhage located in the inferior-temporal macular area

distribution of CME $(p=0.283)$ and $\operatorname{VMT}(p=0.619)$ among the age group categories. Because some patients had undergone cataract surgery (pseudophakic and aphakic eyes), we compared macular abnormality distribution according to lens status. ERM $(p<0.001)$ and $\operatorname{VMT}(p=0.003)$ were significantly more frequent in pseudophakic and aphakic eyes than in unoperated eyes (with clear lens and cataracts) (Fig. 4c; Supplemental Table 5). To eliminate the impact of cataracts on patients' vision, we also analysed the relationship between macular abnormalities and BCVA (logMAR) for RP patients with clear lens, and poor BCVA seemed significantly associated with CME $(p=0.002)$ (Table 2$)$.

\section{Discussion}

To the best of our knowledge, this is the first study to report on the prevalence of ocular abnormalities in a large cohort of RP patients in Western China and to investigate the relationship between BCVA and macular abnormalities detected by OCT. The results showed that the most common ocular abnormalities were cataracts $(43.1 \%)$ and macular abnormalities (59.7\%). In patients with macular abnormalities, CME was significantly associated with poorer visual acuity in RP patients with clear lens.

Macular abnormalities distributed in our study as follows: ERM (51.1\%), CME (18.4\%), VMT (2.4\%), MH (2.3\%), and CNVM (0.05\%). Although the prevalence of 


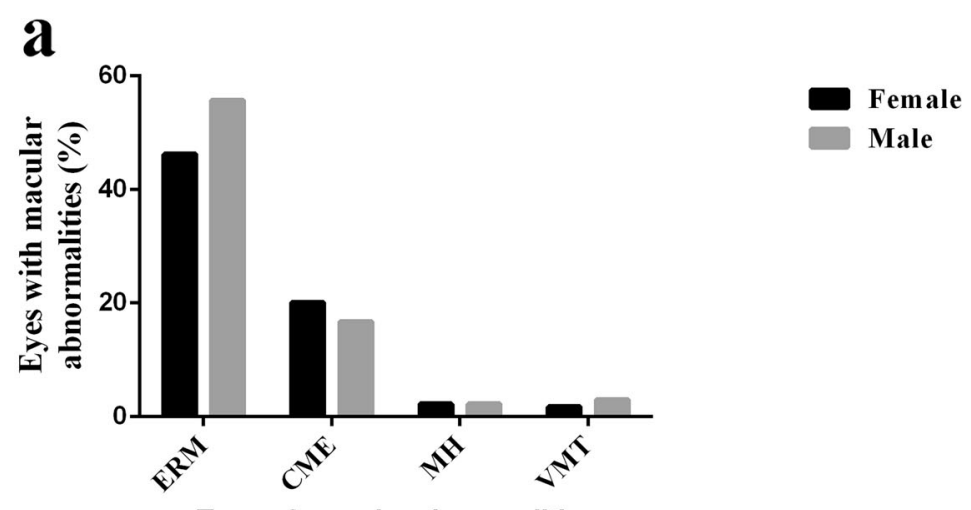

Type of macular abnormalities
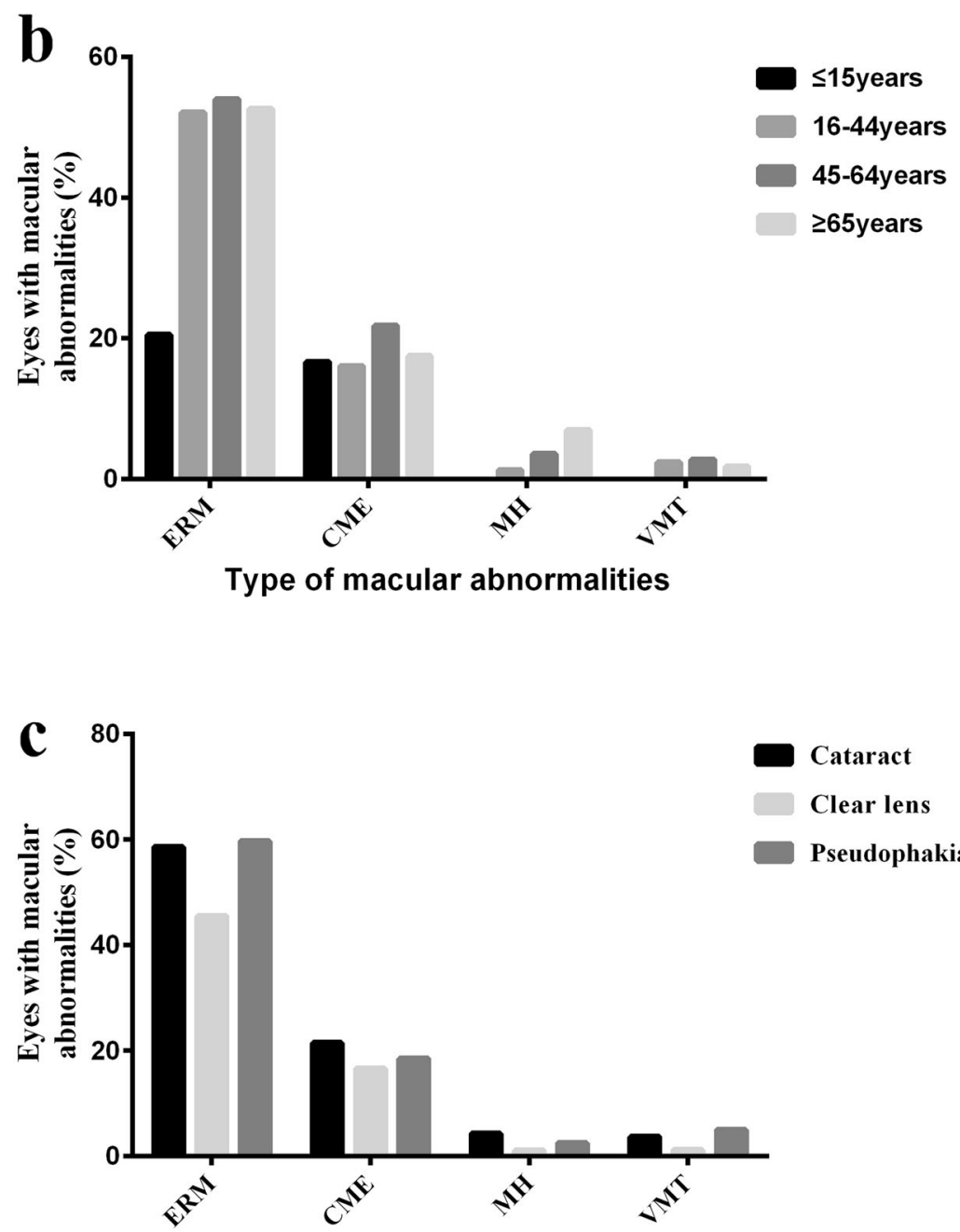

Cataract

Clear lens

Pseudophakia and aphakia

Type of macular abnormalities

Fig. 4 Classification of macular abnormalities in the study cohort of RP patients, stratified by (a) Sex; (b) Age; (c) Lens status. a Bar graph showed no significant differences among the classifications of macular abnormalities and sex (CME: $p=0.193 ; \mathrm{VMT}: p=0.176$; MH: $p=0.383$ ), except for ERM (males: $55.7 \%$; females: $46.3 \% ; p=0.006)$. b This bar graph revealed prevalence of ERM $(p=0.001)$ and MH $(p=0.008)$ were significantly increased with age, while no differences were observed in CME $(p=0.283)$ and VMT ( $p=0.619)$ distributions among age groups. $\mathbf{c}$ ERM ( $p<0.001)$ and VMT ( $p=0.003)$ were significantly more frequent in pseudophakic and aphakic eyes than in unoperated eyes (clear lens and cataracts). Abbreviations: ERM: epiretinal membranes; CME: cystoid macular edema; MH: macular hole; VMT: vitreomacular traction syndrome 
Table 2 Linear regression between macular abnormalities and BCVA (logMAR) in RP patients with clear lens

\begin{tabular}{llllll}
\hline $\begin{array}{l}\text { Variable } \\
\text { (eyes, no.) }\end{array}$ & $\boldsymbol{\beta}$ & $\boldsymbol{t}$ & $\boldsymbol{P}$ value & Lower 95\%Cl & Upper 95\%Cl \\
\hline ERM (368) & 0.020 & 0.406 & 0.685 & -0.076 & 0.115 \\
CME (134) & -0.201 & -3.058 & $0.002^{*}$ & -0.329 & -0.072 \\
MH (9) & 0.194 & 0.842 & 0.400 & -0.258 & 0.646 \\
VMT (10) & 0.167 & 0.756 & 0.450 & -0.266 & 0.599 \\
\hline
\end{tabular}

ERM epiretinal membrane, CME cystoid macular edema, $M H$ macular hole, VMT vitreomacular traction syndrome $\left(^{*}\right)=$ Significant values

macular abnormalities in people of different ages and ethnicities and in regions around the world varies, the frequency of ERM, VMT and MH in the general population has been reported as follows: 9.1\% [15], 1.6-2.4\% [16] and 1.6\%o-2.7\%o [17], respectively. While the distribution of CME and CNVM varies according to age and disease $[16,18]$. ERM has been reported as the second most frequent macular abnormality in RP patients (Table 3). Testa et al's retrospective study of the prevalence of macular abnormalities in Usher syndrome patients [13] and reported a prevalence of $47 \%$. The authors also found the most frequent abnormalities were ERM (19\% of eyes), followed by CME (15.7\%), VMT (14.2\%), and $\mathrm{MH}$ (3.0\%) [13]. However, the prevalence of ERM in our study was much higher (51.1\%) than in previous studies $[4,21,22]$, which may be due to the application of spectral domain-OCT with higher resolution, different genetic backgrounds, and different diagnostic methods. We noted the presence of ERM when even a subtle, hyperreflective lesion adhered to the inner retinal surface, regardless of other abnormalities being present. The mechanisms of ERM formation remain unclear. However, it may include idiopathic preretinal glial cell proliferation, inflammation revealed by an elevated aqueous flare, and chronic macular-vitreous traction [19, 22-24].

In our study, CME was the second most common macular abnormality, which is inconsistent with the results from an Italian population in which Testa et al. investigated macular abnormalities in $581 \mathrm{RP}$ subjects [1], finding that the most frequent abnormality was CME (20.4\% eyes), followed by ERM (15.6\%), VMT (5\%), and $\mathrm{MH}$ (2\%). CME varies from 5.5 to $49 \%$ in RP patients $[4,21]$. The exact mechanism of CME in RP remains unclear; however, it may include the breakdown of the blood-retinal barrier secondary to the degeneration of the RPE and/or the Müller cells, anti-retinal antibodies and traction from ERM and VMT. There is no consensus on the relationship between CME and visual acuity in RP patients [22, 23] . Sandberg et al. found that retinal thinning (due to cell loss) and retinal thickening (due to presumed edema) appeared to be significantly associated with lower visual acuity in RP patients [25]. Yoshida et al. demonstrated that a normal preoperative ellipsoid zone (EZ), also called the inner/outer segment junction (IS/OS), was significantly related to better BCVA in RP patients [26]. Because cataracts and posterior subcapsular cataract (PSCs) were prevalent in RP subjects and were negatively correlated with BCVA, we analysed the relationship between macular abnormalities and BCVA (logMAR) only in eyes with a clear lens. CME appeared to be significantly associated with poor BCVA in our study. The exact relationship between maculopathy and visual acuity requires greater attention in future studies.

CNVM are rare, and until recently, no data have shown the prevalence of CNVM in RP patients. For several years, this information could only be obtained through case reports $[9,14,27]$. In our study, CNVM was observed in only one eye of one female patient (prevalence: approximately $0.09 \%$ ). It has been proposed that photoreceptor cell degeneration and choriocapillaris damage may lead to the formation of CNVM [14].

Although the exact pathophysiology of maculopathy secondary to RP is not fully understood, various pharmacological and surgical treatments for macular abnormalities have been reported [28]. Topical carbonic anhydrase inhibitors (CAI) [29], grid laser

Table 3 Comparison of the prevalence of ocular abnormalities in RP patients with previous studies

\begin{tabular}{|c|c|c|c|c|c|c|c|c|c|}
\hline \multirow[t]{2}{*}{ First author } & \multirow{2}{*}{$\begin{array}{l}\text { Coun- } \\
\text { try }\end{array}$} & \multirow{2}{*}{$\begin{array}{l}\text { Subjects/ } \\
\text { Eyes } \\
\text { (No.) }\end{array}$} & \multicolumn{5}{|c|}{ Macular abnormalities (Eyes / \%) } & \multirow[t]{2}{*}{ Cataract (Eyes/\%) } & \multirow[t]{2}{*}{ Glaucoma (Eyes/\%) } \\
\hline & & & Total & CME & ERM & VMT & MH & & \\
\hline Hajali [6] & USA & $124 / 248$ & & $115 / 46.4$ & & & & & \\
\hline Testa [1] & Italy & $581 / 1161$ & $524 / 45.1$ & $237 / 20.4$ & 181/15.6 & $58 / 5.0$ & $23 / 2.0$ & & \\
\hline Fujiwar-a [19] & Japan & $117 / 206$ & $73 / 35.4$ & & $73 / 35.4$ & & & & \\
\hline Liew [5] & UK & $169 / 338$ & & $172 / 50.9$ & $77 / 22.8$ & & & & \\
\hline Testa [13] & Italy & $134 / 268$ & $126 / 47.0$ & $42 / 15.7$ & $51 / 19.0$ & $38 / 14.2$ & $8 / 3.0$ & & \\
\hline Lee [20] & Korea & $365 / 365$ & & & & & & $175 / 47.9$ & \\
\hline Onakpo-ya OH [2] & Nigeria & $96 / 192$ & $70 / 36.5$ & & & & & $38 / 20$ & $22 / 11.5$ \\
\hline Our study & China & $1065 / 2127$ & $829 / 59.7$ & $255 / 18.4$ & 709/51.1 & $33 / 2.4$ & $32 / 2.3$ & $917 / 43.1$ & $35 / 1.6$ \\
\hline
\end{tabular}


photocoagulation [30], intravitreal therapy with corticosteroids or anti-vascular endothelial growth factor (VEGF) agents [9, 31], and pars plana vitrectomy [32] may be effective for early treatment.

The prevalence of cataract in different age, ethnicities and regions around the world varies. Hashemi et al. conducted a systematic review and meta-analysis of cataract prevalence and found that the age-standardized pooled prevalence estimate (ASPPE) of cataract in populationbased was $17.20 \%$ [33]. Cataracts were the second most common ocular abnormality in our RP patients, and lens opacity developed at a relatively younger age than in the general population. The prevalence $(43.1 \%)$ in our study was similar to the $47.9 \%$ prevalence reported by Lee et al. among Korean patients (Table 3) [20]. PSCs are the most typical morphological abnormalities and occur in $63-83.9 \%$ of RP patients [21, 34, 35]. However, lens status was determined through medical records, and cataract type was unidentifiable in our study. Glaucoma is another ocular abnormality that is prevalent among $\mathrm{RP}$ subjects. There is some evidence to suggest similar genetic backgrounds for glaucoma and RP [12, 36]. Ko et al. reported 3.64-fold greater odds of developing acute angle closure in patients with RP than in the general population [37]. In our study, the prevalence of glaucoma was $2 \%$, which was much lower than the $11.5 \%$ reported by Onakpoya et al. [2] and the $7.5 \%$ reported by Eballe et al. [38], although similar to the prevalence in the general population $(2-3 \%)$ [12]. The reason for the lower rate may be due to our larger sample cohort and our study's retrospective nature.

More than half the RP patients in our study presented with visual acuity deterioration at their first clinical presentation, and the proportion of blindness and low vision as defined by the BCVA were 28.5 and $20.7 \%$, respectively. We defined low vision or blindness according to central visual acuity and did not consider visual field defects. The rates of low vision and blindness in the RP subjects were actually much higher than these results show.

This study had the advantage of having a large sample size, and it assessed various ocular abnormality distributions and visual acuity simultaneously. However, it had several limitations: it was retrospective, and other ocular abnormalities and details, such as corneal nebulae, cataract and glaucoma types, remained unexplored. In addition, most patients had no molecular diagnosis, and we could not sufficiently investigate ocular abnormalities in different genetic subtypes. Further studies, including prospective investigations and studies with more patients with genetic diagnoses, are needed to explore the relationship between the course of RP and BCVA and /or to clarify the relationship between the genetic phenotypes of RP and BCVA.

\section{Conclusion}

The results revealed that ocular abnormalities associated with RP are varied and have high prevalence, especially cataracts and macular abnormalities. Additionally, severe visual impairment was prevalent at the first clinical presentation of RP patients at a single eye hospital in Western China. For macular abnormalities, CME may negatively affect BCVA in RP patients with eyes with clear lens. It is essential to evaluate the macular structure with OCT when accessing RP patients' visual function.

\section{Supplementary Information}

The online version contains supplementary material available at https://doi org/10.1186/s12886-020-01797-z.

Additional file 1: Supplemental Table 1. The BCVA in the study cohort of patients with retinitis pigmentosa stratifying by sex

Additional file 2: Supplemental Table 2. The BCVA in the study cohort of patients with retinitis pigmentosa stratifying by age

Additional file 3: Supplemental Table 3. Classification of macular abnormalities in the study cohort of patients with retinitis pigmentosa stratifying by sex

Additional file 4: Supplemental Table 4. Classification of macular abnormalities in the study cohort of patients with retinitis pigmentosa stratifying by age

Additional file 5: Supplemental Table 5. Classification of macular abnormalities in the study cohort of patients with retinitis pigmentosa stratifying by lens status

\section{Abbreviations}

AD: Autosomic dominant; ASPPE: Age-standardized pooled prevalence estimate; AR: Autosomic recessive; BCVA: Best corrected visual acuity; CAl: Carbonic anhydrase inhibitor; CME: Cystoid macular edema; CNVMs: Choroid neovascularisation membranes; ERMs: Epiretinal membranes; EZ: Ellipsoid zone; ff-ERG: Full-field electroretinogram; IS/OS: Inner/outer segment junction; ISCEV: International Society of Clinical Electrophysiology of Vision; MH: Macular hole; OCT: Optical coherence tomography; PSC: Posterior subcapsular cataract; RP: Retinitis pigmentosa; RPE: Retinal pigment epithelium; VEGF: Vascular endothelial growth factor; VMT: Vitreomacular traction syndrome

\section{Acknowledgements}

We are grateful to Dr. Zhengqin Yin and Dr. Yong Liu, Third Military Medical University (Army Medical University), Chongqing, China, for their help in clinical and statistical data analysis. We thank technicians Minfang Zhang, Sha Li, Bo Liu.

\section{Authors' contributions}

$\mathrm{LT}$ : data collection, interpretation and manuscript preparation, critical revision of the article; YL, ZL, XY, JR and CS: data collection; XM and SL: contributed equally to the study. Investigation design, manuscript preparation and critical revision of manuscript. The authors have read and approved the manuscript and are equally accountable for all aspect of this work.

\section{Funding}

This work was supported by grants from National Nature Science Foundation of China [81974138], National Basic Research Program of China

[2018YFA0107301], and Chongqing Social and Livelihood Science Innovation grant [cstc2017shmsA130100].

Availability of data and materials

Data could be available from the corresponding author by reasonable inquire. 


\section{Ethics approval and consent to participate}

This study was performed according to the Declaration of Helsinki and approved by the Ethics and Research Committee of Southwest Hospital, Army Medical University (KY2020096). Written consent from the patients was not necessary for this non-interventional retrospective chart-review study.

\section{Consent for publication}

Not applicable.

\section{Competing interests}

The author declares no competing interests.

\section{Author details}

'Southwest Eye Hospital/Southwest Hospital, Third Military Medical University (Army Medical University), Chongqing 400038, China. ${ }^{2}$ Key Lab of Visual Damage and Regeneration \& Restoration of Chongqing, Chongqing 400038, China

Received: 21 August 2020 Accepted: 29 December 2020

Published online: 18 January 2021

\section{References}

1. Testa F, Rossi S, Colucci R, Gallo B, Di lorio V, della Corte M, et al. Macular abnormalities in Italian patients with retinitis pigmentosa. Br J Ophthalmol. 2014;98:946-50.

2. Onakpoya OH, Adeoti CO, Oluleye TS, Ajayi IA, Majengbasan T, Olorundare OK, et al. Clinical presentation and visual status of retinitis pigmentosa patients: a multicenter study in southwestern Nigeria. Clin Ophthalmol. 2016:10:1579-83.

3. Vezinaw CM, Fishman GA, McAnany JJ. Visual impairment in retinitis pigmentosa. Retina. 2020;40(8):1630-33.

4. Verbakel SK, van Huet RAC, Boon CJF, den Hollander Al, Collin RWJ, Klaver CCW, et al. Non-syndromic retinitis pigmentosa. Prog Retin Eye Res. 2018;66: 157-86

5. Liew G, Strong S, Bradley P, Severn P, Moore AT, Webster AR, et al. Prevalence of cystoid macular oedema, epiretinal membrane and cataract in retinitis pigmentosa. Br J Ophthalmol. 2019:103:1163-6.

6. Hajali M, Fishman GA, Anderson RJ. The prevalence of cystoid macular oedema in retinitis pigmentosa patients determined by optical coherence tomography. Br J Ophthalmol. 2008;92:1065-8.

7. Hajali M, Fishman GA. The prevalence of cystoid macular oedema on optical coherence tomography in retinitis pigmentosa patients without cystic changes on fundus examination. Eye (Lond). 2009;23:915-9.

8. Liew G, Moore AT, Bradley PD, Webster AR, Michaelides M, et al. Factors associated with visual acuity in patients with cystoid macular oedema and retinitis Pigmentosa. Ophthalmic Epidemiol. 2018;25:183-6.

9. Miyata M, Oishi A, Oishi M, Hasegawa T, Ikeda HO, Tsujikawa A. Long-term efficacy and safety of anti-VEGF therapy in retinitis pigmentosa: a case report. BMC Ophthalmol. 2018;18:248.

10. Schulze-Bonsel K, Feltgen N, Burau H, Hansen L, Bach M. Visual acuities "hand motion" and "counting fingers" can be quantified with the Freiburg visual acuity test. Invest Ophthalmol Vis Sci. 2006:47:1236-40.

11. McCulloch DL, Marmor MF, Brigell MG, Hamilton R, Holder GE, Tzekov R, et al. ISCEV standard for full-field clinical electroretinography (2015 update). Doc Ophthalmol. 2015;130:1-12.

12. Liu X, Li J, Lin S, Xiao X, Luo J, Wei W, et al. Evaluation of the genetic association between early-onset primary angle-closure glaucoma and retinitis pigmentosa. Exp Eye Res. 2020;197:108118.

13. Testa F, Melillo P, Rossi S, Marcelli V, de Benedictis A, Colucci R, et al. Prevalence of macular abnormalities assessed by optical coherence tomography in patients with usher syndrome. Ophthalmic Genet. 2018;39: 17-21.

14. Sayadi J, Miere A, Souied EH, et al. Type 3 neovascularization associated with retinitis Pigmentosa. Case REP Ophthalmol. 2017:8:245-9.

15. Xiao W, Chen X, Yan W, Zhu Z, He M. Prevalence and risk factors of epiretinal membranes: a systematic review and meta-analysis of populationbased studies. BMJ Open. 2017;7(9):e014644

16. Meuer SM, Myers CE, Klein BE, Swift MK, Huang Y, Gangaputra S, et al. The epidemiology of vitreoretinal interface abnormalities as detected by spectral-domain optical coherence tomography: the beaver dam eye study. Ophthalmology. 2015;122(4):787-95.
17. McCannel CA, Ensminger JL, Diehl NN, Hodge DN. Population based incidence of macular holes. Ophthalmology. 2009;116(7):1366-9.

18. Yang MC, Chen YP, Tan EC, Leteneux C, Chang E, Chu CH, et al. Epidemiology, treatment pattern and health care utilization of myopic choroidal neovascularization: a population based study. Jpn J Ophthalmol. 2017;61(2):159-68.

19. Fujiwara K, Ikeda Y, Murakami Y, Nakatake S, Tachibana T, Yoshida N, et al. Association between aqueous flare and Epiretinal membrane in retinitis Pigmentosa. Invest Ophthalmol Vis Sci. 2016;57:4282-6.

20. Lee SH, Yu HG, Seo JM, Moon SW, Moon JW, Kim SJ, et al. Hereditary and clinical features of retinitis Pigmentosa in Koreans. J Korean Med Sci. 2010; 25:918-23.

21. Hartong DT, Berson EL, Dryja TP. Retinitis pigmentosa. Lancet. 2006;368: 1795-809.

22. Strong S, Liew G, Michaelides M. Retinitis pigmentosa-associated cystoid macular oedema: pathogenesis and avenues of intervention. $\mathrm{Br} J$ Ophthalmol. 2017;101:31-7.

23. Liu G, Liu X, Li H, Du Q, Wang F. Optical coherence tomographic analysis of retina in retinitis Pigmentosa patients. Ophthalmic Res. 2016;56:111-22.

24. Al Rashaed S, Khan AO, Nowilaty SR, Edward DP, Kozak I. Spectral-domain optical coherence tomography reveals prelaminar membranes in optic nerve head pallor in eyes with retinitis pigmentosa. Graefes Arch Clin Exp Ophthalmol. 2016;254(1):77-81

25. Sandberg MA, Brockhurst RJ, Gaudio AR, Berson EL. The association between visual acuity and central retinal thickness in retinitis Pigmentosa. Invest Ophthalmol Vis Sci. 2005:46:3349-54.

26. Yoshida N, Ikeda Y, Murakami Y, Nakatake S, Fujiwara K, Notomi S, et al. Factors affecting visual acuity after cataract surgery in patients with retinitis Pigmentosa. Ophthalmology. 2015;122:903-8.

27. Battaglia Parodi M, De Benedetto U, Knutsson KA, Scotti F, Librando A, Bandello F, et al. Juxtafoveal Choroidal neovascularization associated with retinitis Pigmentosa treated with Intravitreal Bevacizumab. J Ocul Pharmacol. 2012:28:202-4.

28. Bakthavatchalam M, Lai FHP, Rong SS, Ng DS, Brelen ME. Treatment of cystoid macular edema secondary to retinitis pigmentosa: a systematic review. Surv Ophthalmol. 2017;63(3):329-39.

29. Grover S, Fishman GA, Fiscella RG, Adelman AE. Efficacy of dorzolamide hydrochloride in the management of chronic cystoid macular edema in patients with retinitis pigmentosa. Retina. 1997;17(3):222-31.

30. Newsome DA, Blacharski PA. Grid photocoagulation for macular edema in patients with retinitis pigmentosa. Am J Ophthalmol. 1987;103(2):161-6

31. Artunay O, Yuzbasioglu E, Rasier R, Sengul A, Bahcecioglu H. Intravitreal Ranibizumab in the treatment of cystoid macular edema associated with retinitis Pigmentosa. J Ocul Pharmacol Ther. 2009;25(6):545-50.

32. García-Arumí J, Martinez V, Sararols L, Corcostegui B. Vitreoretinal surgery for cystoid macular edema associated with retinitis Pigmentosa. Ophthalmology. 2003;110(6):1164-9.

33. Hashemi H, Pakzad R, Yekta A, Aghamirsalim M, Pakbin M, Ramin S, et al. Global and regional prevalence of age-related cataract: a comprehensive systematic review and meta-analysis. Eye (Lond). 2020;34(8):1357-70.

34. Dikopf MS, Chow CC, Mieler WF, Tu EY. Cataract extraction outcomes and the prevalence of Zonular insufficiency in retinitis Pigmentosa. Am J Ophthalmol. 2013;156:82-8.

35. Fujiwara K, Ikeda Y, Murakami Y, Funatsu J, Nakatake S, Tachibana T, et al. Risk factors for posterior subcapsular cataract in retinitis Pigmentosa. Invest Ophthalmol Vis Sci. 2017;58:2534-7

36. Fernández-Martínez L, Letteboer S, Mardin CY, Weisschuh N, Gramer E, Weber BH, et al. Evidence for RPGRIP1 gene as risk factor for primary open angle glaucoma. Eur J Hum Genet. 2011;19:445-51.

37. Ko YC, Liu CJ, Hwang DK, Chen TJ, Liu CJ. Increased risk of acute angle closure in retinitis Pigmentosa: a population-based case-control study. PLoS One. 2014;9:e107660.

38. Eballe AO, Koki G, Emche CB, Bella LA, Kouam JM, Melong J. Blindness and visual impairment in retinitis pigmentosa: a Cameroonian hospital-based study. Clin Ophthalmol. 2010;4:661-5.

\section{Publisher's Note}

Springer Nature remains neutral with regard to jurisdictional claims in published maps and institutional affiliations. 